\title{
TRAUMATIC ACUTE PANCREATITIS AS A CAUSE OF LARGE BOWEL OBSTRUCTION
}

\author{
R. F. Bolam, M.B., B.S. \\ Surgical Registrar, Regional Thoracic Unit, Brook General Hospital, Woolwich \\ Formerly Resident Surgical Officer, St. Alfege's Hospital, Greenwich
}

Acute pancreatitis occurring as a result of trauma, either from a penetrating wound or a subcutaneous injury is sufficiently well known to merit discussion in text books. On the other hand intestinal obstruction developing as a direct result of pancreatitis is excessively rare, if one excludes those cases of paralytic ileus secondary to a severe suppurative pancreatitis of which the outcome is almost always fatal. Miln and Barclay (1952) described two cases of colonic obstruction sited at the splenic flexure treated initially by caecostomy. Further laparotomies at later dates revealed that acute pancreatitis had been the initiating cause. These authors found three other similar cases in the literature.

\section{Case Report}

A young man of 20 was admitted to hospital on March 5, 1958, having driven his motorcycle into a stationary lorry. He was wearing a crash helmet. On examination he was semi-comatose, responding only to painful stimuli. Pulse, regular and full; rate $80 / \mathrm{min}$., B.P. $126 / 80$. He was bleeding from his right ear and nose and had two cuts on his forehead. There was a large haematoma over the left frontal bone and swelling of the left side of the neck. No other injuries were detected and no abnormal neurological signs were demonstrated. At this time a note was made that the abdomen was held rigid. Although no fractures were seen on X-ray examination, it was considered that the base of the skull had been fractured.

The patient remained in this semi-comatose condition for several days regaining consciousness by March 9, after which he appeared to recover normally. However on March 3 I he complained that over the past two or three days he had vomited after eating. He was found to be tender in the epigastrium, had a temperature of $100^{\circ}$ and a pulse rate of 90 . On the following day the abdomen was distended, with tenderness in the epigastrium and both iliac fossae. The temper- ature was normal but the pulse rate had risen to I20/min. There had been absolute constipation for two days. A plain X-ray of the abdomen in the erect position showed multiple fluid level suggestive of a large bowel obstruction.

Gastric aspiration and intravenous fluid therapy was instituted and then a laparotomy was performed through a right paramedian incision. Pus. was found in the peritoneal cavity. The appendix, stomach and duodenum were normal. There were numerous coils of distended small bowel and the large bowel was distended from the caecum as far as the splenic flexure where it disappeared into a large mass comprised of omentum, pancreas and spleen. Beyond this the descending colon, pelvic colon and rectum were collapsed. The pancreas was grossly enlarged with areas of fat necrosis and haemorrhage. Fat necroses were apparent in the omentum and transverse mesocolon. After peritoneal toilet a transverse loop colostomy was performed proximal to the obstructing lesion, and the abdomen closed with drainage. He was given a course of Tetracyclin and the abdominal condition resolved over the next few days, but a week after the operation he was found to have signs of a left pleural effusion. He looked ill and toxic with a swinging temperature up to $\mathrm{ror}^{\circ}$ and a pulse rate of $130.24 \mathrm{oz}$. of greenish yellow fluid were withdrawn by aspiration from the left pleural cavity, and this grew B. Coli on culture. This empyema was treated by repeated aspirations and the instillation of streptomycin solution into the cavity for the following two weeks.

On May 9, a barium enema was performed which showed that there was now no obstruction in the large bowel. As the chest condition had resolved completely by this time a further operation was carried out on May 18 to close the colostomy, abdominal exploration not being performed. Following this normal bowel actions started and he was finally discharged from hospital on May 28. He has remained symptom free since. 


\section{Comment}

In those cases suffering from severe injury to the abdomen laparotomy is likely to be performed even if traumatic pancreatitis is suspected, as injury to other organs cannot be excluded. With less severe subcutaneous injuries the correct diagnosis may be made if trauma, as an aetiological factor in some instances of acute pancreatitis, is borne in mind. The serum amylase estimation may be helpful in this direction, and should be performed in the early stages of all cases of abdominal trauma if injury to the pancreas is suspected. A normal value, if estimated for the first time some days after injury, cannot rule out the possibility of pancreatic injury as even a very high level at the onset of a pancreatitis tends to fall to normal within a few days. Furthermore the amylase level is no criterion of the severity of the affection of the pancreas. But in the present case the picture of cerebral contusion obscured the concomitant pancreatic pathology. It is assumed that the pancreas was injured at the time of the accident; possibly only a few acini were ruptured and little haemorrhage into the gland substance took place but the resulting liberation of enzyme was sufficient to initiate the changes of acute pancreatitis. Presumably if the patient had been conscious symptoms and signs of pancreatitis would have beer present, although it is not rare to find, especially in older patients, that pain and tenderness have been minimal even when a later laparotomy has revealed extensive pancreatic disease. At any rate it would seem likely that when the patient recovered consciousness there would be no acute abdominal symptoms; this is in agreement with the fact that in the majority of cases of acute pancreatitis the symptoms subside after a few days.

But even after the disappearance of all symptoms and signs pathological changes continue in the pancreas and surrounding tissues depending on the severity of the initial lesion. This can rarely; so it seems, lead to a large bowel obstruction, and, occurring after an interval of time might not be considered to be related to the original disease, if in fact that had been recognised. The site of obstruction at the splenic flexure is dependent upon the anatomical arrangement. This flexure is the most fixed part of the colon, it is acutely flexed and it is in relationship to the tail of the pancreas while the left border of the greater omentum crosses the transverse colon in this region. Thus this part of the colon is more likely to become involved in the inflammatory process when there is extension into the greater omentum and transverse mesocolon. Although extensive fat necrosis and fibrosis in these structures and in the appendices epiploicae may lead to constriction of the bowel it is likely that oedema of the bowel $\stackrel{\varrho}{\square}$ wall, from involvement in the inflammatory mass, $\frac{\mathbb{D}}{\mathrm{Q}}$ is the final obstructing agent. When the in $-\frac{2}{c}$ flammation subsides with disappearance of the oedema the bowel lumen becomes patent again.

If this is the pathological process treatment iso simple. Provided that the diagnosis is made ato laparotomy nothing more is required surgically $\overline{\bar{c}}$. than a proximal colostomy to relieve the obstruc- $\mathbb{0}$ tion combined with suitable antibiotics ando correction of any electrolytic deficiency. After $a_{-}^{\infty}$ sufficient interval to allow the inflammation to. subside, and afier restitution of the bowel lumen $\overrightarrow{\vec{\omega}}$ has been demonstrated by barium enema, theo continuity of the transverse colon can be re-80 established. Treatment by excision of the spleen, 3 . tail of pancreas and affected part of colon andiw omentum as recommended by Remington, Mayo:

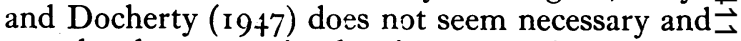
may be dangerous in that it may evoke a recru-in descence of the pancreatitis with the possibility of a fatal outcome. If there is residual narrowing of the bowel lumen a short circuit operation mayc be preferable to an extensive resection, especially in the older patient. Persistent, complete occlusiond of the lumen will probably mean that the originak diagnosis was at fault and that the true cause $8 \mathrm{f}_{6}$ the obstruction is a carcinoma, which may hageo caused a localized pancreatitis by infiltrating i the neighbouring pancreas.

One aspect of this case remains to be discussed, the occurrence of an empyema in the left pleuralo cavity. A left pleural effusion may appear as å complication during the course of acute pancreatitis. (Coffey, 1952.) It is probable that thiso patient developed a small effusion which was not recognized initially and that this became infected secondarily by B. Coli from the peritoneal cavity.

\section{Summary}

A case of large bowel obstruction resulting from traumatic pancreatitis is described. The difficultyo of diagnosis of an intra abdominal lesion in the presence of a concomitant head injury is discussed $\mathrm{O}$ Relief of the obstruction by the simplest method? namely a colostomy, is recommended.

\section{Acknowledgmen}

I wish to thank Mr. M. G. Eldin for permission to report this case.

\section{REFERENCES}

COFFEY, R. J. (1952), Ann. Surg., 135, 7 1 5. MILN, D. C., and BARCLAY, T. H. C. (1952), Lancet, ii, 168 REMINGTON, J. H., MAYO, C. W., and DOCHERTY, M. BS (1947), Proc. Mayo Clinic, 22, 260. 